\title{
In situ nucleoprotein structure at the SV40 major late promoter: melted and wrapped DNA flank the start site
}

\author{
Li Zhang and Jay D. Gralla \\ Department of Chemistry and Biochemistry, and the Molecular Biology Institute, University of California, Los Angeles, \\ California 90024-1569 USA
}

\begin{abstract}
New in situ probing methods have been developed and used to probe the nucleoprotein structures at the SV40 major late promoter in infected monkey cells. The region that contains the three proximal transcription elements was probed with DNase I and micrococcal nuclease in transcriptionally active, permeabilized cells, and with the single-strand selective reagent $\mathrm{KMnO}_{4}$ in intact cells. The downstream element is included in a region of enhanced DNase I reactivity at 10- to 11-bp intervals for $\sim 140 \mathrm{bp}$, presumably because of DNA wrapping around a specifically positioned nucleosome particle. The two other proximal DNA elements appear to be mostly melted, with a protecting factor bound primarily to the template DNA strand. The protecting factor directly borders the wrapped particle. These observations provide an initial description of parts of the biological transcription machinery and suggest that the SV40 major late promoter elements are part of a higher order nucleoprotein complex that involves wrapped and melted DNA.
\end{abstract}

[Key Words: Promoters; DNA; nucleoprotein; SV40; transcription factors]

Received June 26, 1989; revised version accepted September 4, 1989.

Simian virus 40 (SV40) contains two transcription units in its small 5243-bp genome. Both the early and late promoters use the host monkey cell RNA polymerase II and associated machinery, making the small virus a convenient model for studying mammalian transcription mechanisms. The SV40 controlling elements are located in a compact 400-nucleotide region and bind many mammalian regulatory proteins. The early unit codes for small and large $T$ antigens, which prepare the virus for DNA replication and late transcription. The late genes are activated after the onset of DNA replication and code principally for viral coat proteins. The initiation sites of late mRNA are heterogeneous; the major late start site is at nucleotide 325 , which accounts for the large majority of the transcripts. The SV40 major late promoter, which is responsible for the production of this mRNA, includes three contiguous proximal regulatory elements (Brady et al. 1982; Ayer and Dynan 1988): the first extends $31 \mathrm{bp}$ upstream of the transcription start site, the second is at the start site, and the third is centered $28 \mathrm{bp}$ downstream. The roles of these elements in assembling an active transcription complex are unknown.

The SV40 major late promoter resembles other polymerase II promoters in that it contains multiple proximal elements, although the SV40 elements themselves are not common. Many DNA transcription regulatory elements are capable of binding more than one protein (e.g., see Dorn et al. 1987). This observation, coupled with the unknown role of general chromatin-associated proteins, makes it difficult to know which components are required for the physiologically appropriate reconstruction of transcription systems. In part, because of this multiplicity of factors and the lack of knowledge concerning which factors are appropriate, the anatomy of the physiological polymerase II transcription complex remains relatively obscure, although there has been progress in defining minimal in vitro assembly pathways (see Buratowski et al. 1989). Therefore, we are developing new methods that allow the physiologically appropriate complexes to be probed in situ, using the SV40 regulatory region as the initial test system.

Several methods have been applied to study nucleoprotein complexes in situ. Previously, we used DNase I in conjunction with primer-extension detection methods (Gralla 1985) to probe the SV40 GC-box region in isolated nuclei (Buchanan and Gralla 1987). However, because isolated CV-1 and other nuclei are usually incapable of supporting transcription initiation, we sought alternative methods that would allow probing of the active transcription machinery. Dimethyl sulfate has been used as a chemical probe in certain cells (see Ephrussi et al. 1985|, but its ability to penetrate nucleoprotein complexes and its strong preference for guanines have limited its utility. In this study, we have developed new procedures for probing cells that significantly broaden the 
range of interactions that may be seen (see Fig. 1). The results show selective and unanticipated in situ interactions with the major late promoter elements.

\section{Results}

Nucleases have long been used to probe DNA-protein interactions in vitro and in isolated nuclei /Galas and Schmitz 1978; Piette et al. 1986; Buchanan and Gralla 1987). Because nucleases are too large to enter cells, they have not been used to probe active transcription complexes in situ. Some time ago, Contreras and Fiers (1981) showed that lysolecithin permeabilization of SV40-infected CV-1 cells allowed entry of radiolabeled ATP that was incorporated into the 5 '-end of the major late SV40 mRNA as a result of transcription initiation. We used this permeabilization treatment to attempt to introduce DNase I under similar conditions, with the intention of probing the intact SV40 transcription machinery.

\section{Probing permeabilized cells with DNase I}

The procedure for probing SV40 DNA in infected cells is outlined briefly in Figure 1. Infected CV-1 cell monolayers were permeabilized by adding $0.05 \%$ lysolecithin for $1 \mathrm{~min}$ at $37^{\circ} \mathrm{C}$, and the lysolecithin solution was subsequently aspirated off the plates. Then the cells were rinsed to remove the residual lysolecithin and treated with DNase I for $2 \mathrm{~min}$ at $37^{\circ} \mathrm{C}$. The enzyme solution was aspirated off the plates and Hirt lysis solution was added immediately to quench the residual enzyme activity. Finally, the SV40 DNA was purified as described previously (Buchanan and Gralla 1987), and the sites of DNase-induced nicking in situ were analyzed by primer-extension procedures (Gralla 1985). Primers used in this study are shown by arrows at the bottom of Figure 1 ; the number indicates the number of the $5^{\prime} \mathrm{nu}$ cleotide to which they hybridize.

In this initial test of the introduction of DNase I into permeabilized cells, the nicked denatured DNA samples were probed with two different primers. Primer 404 hybridizes downstream from the major late promoter, and its extension products define the in situ DNase cleavage sites in the promoter region (Fig. 2A). For comparison, primer 2556 was also hybridized and extended to reveal the cleavage pattern near the SV40 termination region (Fig. 2B). For each experiment, samples were probed that resulted from two different amounts of added enzyme. Two similar control experiments were run, where naked DNA was digested with DNase in vitro, and these samples were probed by these same two primers. The amount of DNase required to reveal a digestion pattern was very high in the in situ experiments, approximately $10^{4}$ times the amount used to digest naked DNA and 10 times the amount used previously to probe nuclei (Buchanan and Gralla 1987). Although lysolecithin does facilitate entry of the enzyme into the nucleus, the requirement for much more nuclease suggests that the process is relatively inefficient.

When the in vitro and in situ DNase I digestion pat-
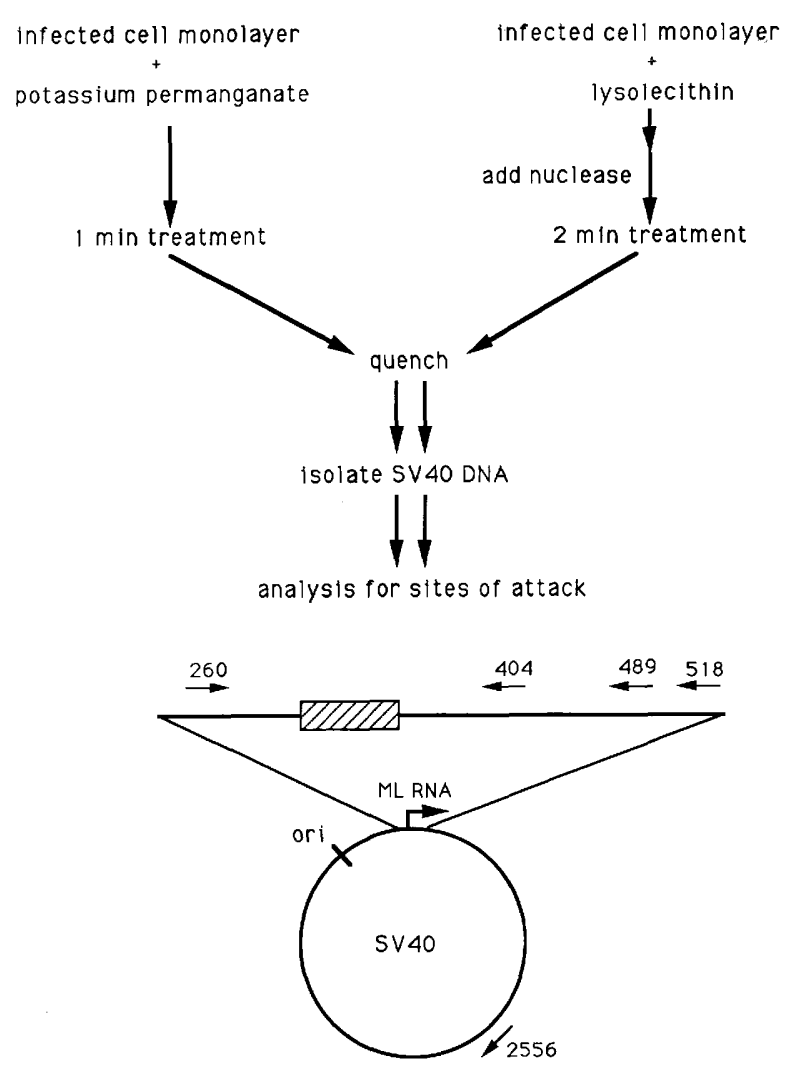

Figure 1. Brief outline of the procedure for the treatment of SV40-infected intact and permeabilized cells with $\mathrm{KMnO}_{4}$ DNase I and MNase. Primers used to probe DNA are shown below. The major late start site is shown, and the proximal late transcription elements are marked by the hatched box.

terns are compared, both similarities and differences are readily observable. With respect to the termination region, these are best seen by comparing lane 1 (in situ) with lane 2 (in vitro) of Figure 2B. Because the overall patterns are reasonably similar, it is possible to identify individual nucleotides, which are either protected or made hyperreactive to DNase attack in situ. This region of the genome is known to contain nucleosomes (Griffith 1975; Shelton et al. 1978), but because the regulatory DNA sequences are elsewhere, specific protein binding has not been postulated, consistent with the lack of long protected regions in lane 1. Nucleosomes positioned randomly over this region would not lead to regular pattern changes, but rather scattered hyperreactive and protected positions, as is observed. A hyperstable nucleosome is known to form over this region in in vitro reconstituted chromatin (Poljak and Gralla 1987). If this occurred in vivo at a specific position it would lead to DNase I hyperreactivity at approximately 10- to 11-bp intervals (Simpson and Stafford 1983; Drew and Travers 1985). Some hypersites do appear in the pattern (lane 1), but these are not positioned at appropriate periodic intervals. Thus the pattern with nonperiodic hypersites probably indicates a mixture of nucleosomes at different sequence positions or, perhaps, other unknown aspects of chromatin structure. 

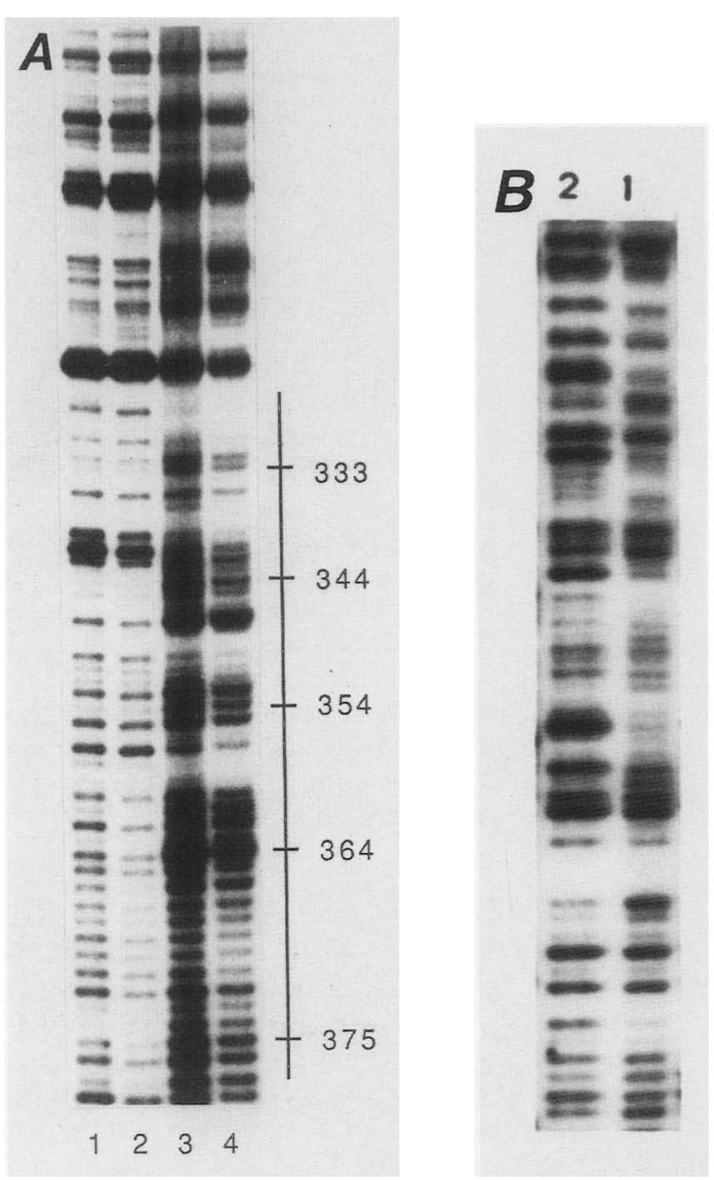

Figure 2. (A) DNase I digestion pattern of the major late promoter region in lysolecithin-permeabilized cells. Permeabilized SV40-infected CV-1 cells were treated with $5 \times 10^{-2} \mathrm{mg} / \mathrm{ml}$ (lane 3), or $10^{-1} \mathrm{mg} / \mathrm{ml}$ (lane 4) DNase I in solution $\mathrm{B}$ at $37^{\circ} \mathrm{C}$, and SV40 DNA was purified and probed with primer 404. Each in vivo lane was derived by DNase I digestion of permeabilized cells from one-half of a $10-\mathrm{cm}$-diameter plate at $40 \mathrm{hr}$ post-infection. As controls, purified SV40 DNA was digested with DNase I [ $5 \times 10^{-6} \mathrm{mg} / \mathrm{ml}$ (lane 2), or $10^{-5}$ (lane 1$\left.)\right]$ and probed with the same primer. $(B)$ DNase I digestion pattern near the termination region by use of primer 2556 ; lane 1 , in situ DNase I digestion pattern of SV40 DNA in permeabilized cells. Lane 2, in vitro naked DNase I digestion pattern.

By contrast, the patterns near the SV40 major late promoter are similar only in the upper portion of the autoradiogram and diverge in the lower portion /cf. lanes 2 and 4 of Fig. 2A). Certain regions are very strongly hyperreactive to DNase in situ, and these appear at approximately 10 - to 11 -bp intervals. These occur at positions corresponding to nucleotides $333,344,354,364$, and 375 (lanes 3 and 4 of Fig. 2A). This signature pattern of DNase I hyperreactive cleavages is absent from the samples obtained by the cleavage of naked DNA in vitro, which exhibit cutting with no obvious periodicity (lanes 1 and 2 of Fig. 2A).

Periodicity of this type was described originally as a property of specifically positioned nucleosomes, but has since been found to occur along DNA which is wrapped or curved as part of certain nonnucleosomal structures (Drew and Travers 1985; Sawadogo and Roeder 1985; Hochschild and Ptashne 1986). Periodic patterns associated with other promoters (Sawadogo and Roeder 1985; Zinn and Maniatis 1986) generally occur over a limited region, whereas that of the nucleosome continues over a very large region, perhaps $120-160 \mathrm{bp}$ (Simpson and Stafford 1983; Drew and Travers 1985). Therefore, two new primers (489 and 518) were used to probe the distal region to observe the possible extension of the periodic pattern. Figure 3, A and B shows densitometric scans of autoradiographs of the DNase I digestion pattern obtained with primer 489 (A and B are aligned exactly according to nucleotide position). On comparison of the in situ pattern (Fig. 3B) with the in vitro pattern (Fig. 3A), it is clear that the periodicity is extended in situ (peaks shown by arrows in 3B). The control samples digested in vitro show no periodic digestion and mostly very weak signals are observed at positions corresponding to strong signals in situ (Fig. 3A, arrows). The in situ pattern shows six peaks at nucleotides 465,455 , $444,434,425$, and 415 , which are in the same periodic register as the peaks nearer to the transcription start site (Fig. 2A). Analysis of results obtained with primer 518, which is able to probe the region up to nucleotide 500, did not reveal periodicity beyond nucleotide 465 (data not shown).

The observation of enhanced DNase I reactivity at 10 to 11 -bp intervals over a nucleosome size region (nucleotide 333 to 465 ) strongly suggests that a specifically positioned nucleosome is present. The lack of strong hyperreactivity between nucleotides 385 and 405 would be consistent with a nucleosome center near position 395 ; the center and the sites around it constitute the longest region of weak DNase I cleavage in nucleosomes (Lutter 1978). These results, however, do not imply that nucleosomes are absent from other regions that do not show periodicity. As mentioned above, nucleosomes are likely present elsewhere, but their relatively random positioning would yield overlapping patterns in which the periodicity associated with each position was masked (Chao et al. 1979).

The particle giving rise to this nucleosome pattern is positioned just downstream from the initiation site for late RNA synthesis at nucleotide 325 . It covers one of the three known proximal promoter elements that is centered near nucleotide 353 (Ayer and Dynan 1988). However, the DNase I cleavage pattern over the two upstream proximal promoter elements is not very informative. This is partly because there are relatively few DNase I cleavage sites in this region even in the in vitro control lane. To probe these elements we turned to the use of another nuclease with a different mode of DNA recognition and cleavage.

\section{Probing with micrococcal nuclease}

Micrococcal nuclease (MNase) is a small enzyme $\left(M_{\mathrm{r}}=16 \mathrm{kD}\right)$ with a DNA recognition mode that differs 


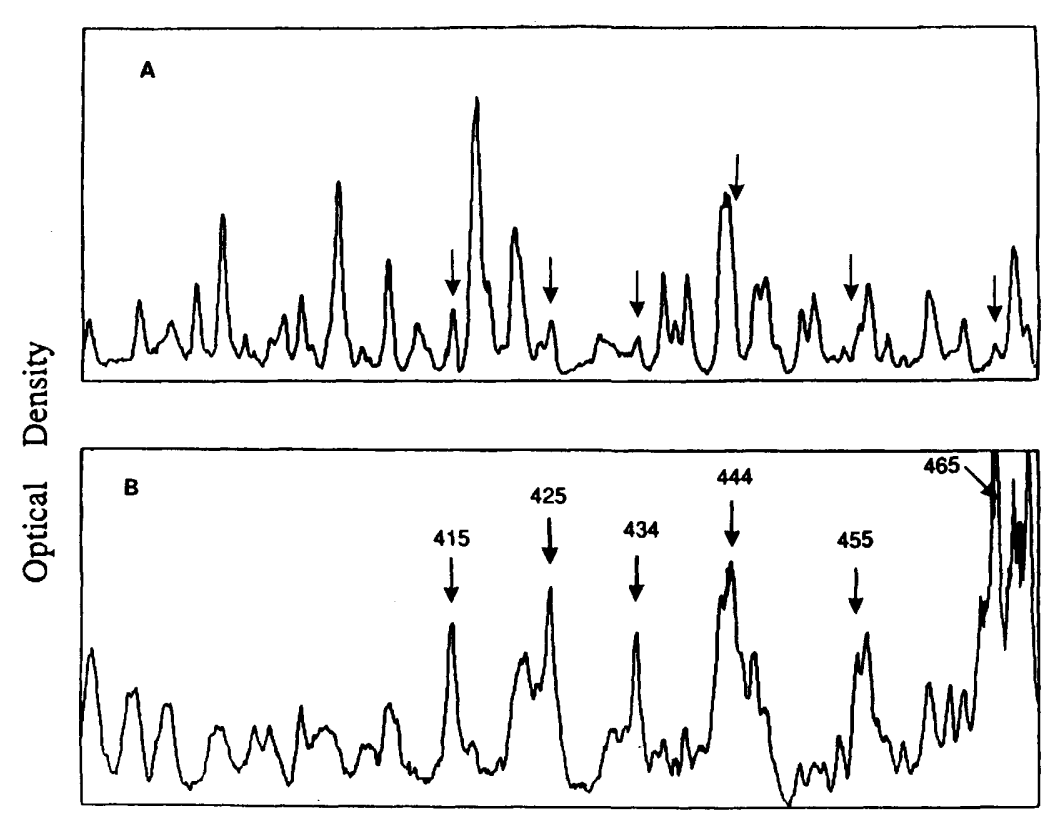

Position
Figure 3. $(A)$ Densitometric scan of DNase I digestion pattern of in vitro naked DNA from nucleotide 470 to 400 . The primer used for $A$ and $B$ was primer 489 , which hybridizes to the early mRNA template strand from nucleotide 508 to 489. $(B)$ Scans of permeabilized cell DNase I digestion pattern of the same region. Scans $A$ and $B$ are from parallel lanes on the same gel and they are aligned exactly according to nucleotide position. The positions of the periodic peaks are shown by arrows with their SV40 nucleotide number noted. The same positions are marked with arrows in the in vitro pattern shown in $A$. significantly from that of DNase I. Whereas DNase I recognizes and binds the DNA minor groove, MNase recognizes and cleaves the DNA backbone directly (Dingwall et al. 1981; Dieters et al. 1982). Cleavage of adenine and thymine positions is favored. In general, the enzyme prefers exposed regions and therefore cuts singlestranded DNA much more rapidly than double-stranded DNA (Cockell et al. 1983; Drew 1984). MNase cleavage is inhibited by bound proteins and can be used both as a footprinting reagent to detect backbone-binding proteins and as a sensor of exposed backbone positions (Zhang and Gralla 1989).

MNase was introduced into permeabilized cells as described above for DNase I (see Fig. 1), and the resultant nicked DNA was purified, denatured, and probed with primer 260. The resulting extension pattern (Fig. 4A, lanes 1 and 2) displays the cleavages within the three proximal promoter elements. For purpose of comparison, naked DNA was cleaved in vitro with MNase and probed in parallel (lanes 3 and 4). The figure shows that over much of the region probed, the in vitro and the in situ patterns are virtually identical. The principal exception is the region from nucleotide 316 to nucleotide 330 , which corresponds to the central element overlapping the transcription start site at nucleotide 325 . In this region, many of the MNase cleavages in situ are significantly weaker than the cleavages in vitro (compare in situ lane 2 with in vitro lane 3 ). The extent of protection suggests that the majority, if not all, of the templates probed contain a factor that protects this element from attack by MNase.

This region could not be probed carefully in the prior DNase I experiments because few bands were present even in the naked DNA control; the single major DNase I band present in vitro (nucleotide 327), however, was protected against DNase attack in situ (Fig. 2A, lane 2 versus lane 3 , above the marked nucleotide 333 ). The
MNase cleavage pattern does not show selective periodicity in situ as was observed with DNase I. The DNase I periodic pattern suggested a positioned nucleosome, but the MNase cleavage pattern is uninformative in this regard because periodic MNase cleavage is not a reliable marker of a positioned nucleosome (see McGhee and Felsenfeld 1983).

Next, we used primer 404 to probe cleavages on the other DNA strand in this same region. Initial low resolution results showed that the differences in pattern between in vitro and in situ samples were primarily within the proximal transcription elements (data not shown). A high resolution probing of this region is shown in Figure $4 \mathrm{~B}$. Beyond nucleotide 340 , the in situ pattern llane 2 of Fig. $4 \mathrm{~B}$ ) is similar to the in vitro cleavage pattern (lane 1), except that the in vitro lane is somewhat lighter because slightly less DNA was loaded. Upstream of this point, however, the patterns diverge significantly. Many hyperactive cleavages are seen in the in situ lane 2 . The strongest of these flank the transcription start site. The start site, however, is protected from attack (note the only position where lane 2 is significantly lighter than lane 1).

The results of probing both strands point to an in situ interaction of a factor over the central proximal transcription element, just upstream from the specifically positioned nucleosome. One strand of the element has the DNA backbone protected from cleavage, and the other strand has the backbone selectively exposed to attack, except near the start site, which is protected. The exposure is indicated by the selective hyperreactivity to MNase attack in situ. Because MNase selectively attacks single-stranded DNA, we wondered if this hyperreactivity reflected DNA melting around the transcription start site. Such melting occurs in bacterial transcription complexes and can be detected by the single-strand selective reagent $\mathrm{KMnO}_{4}$ (Sasse-Dwight 

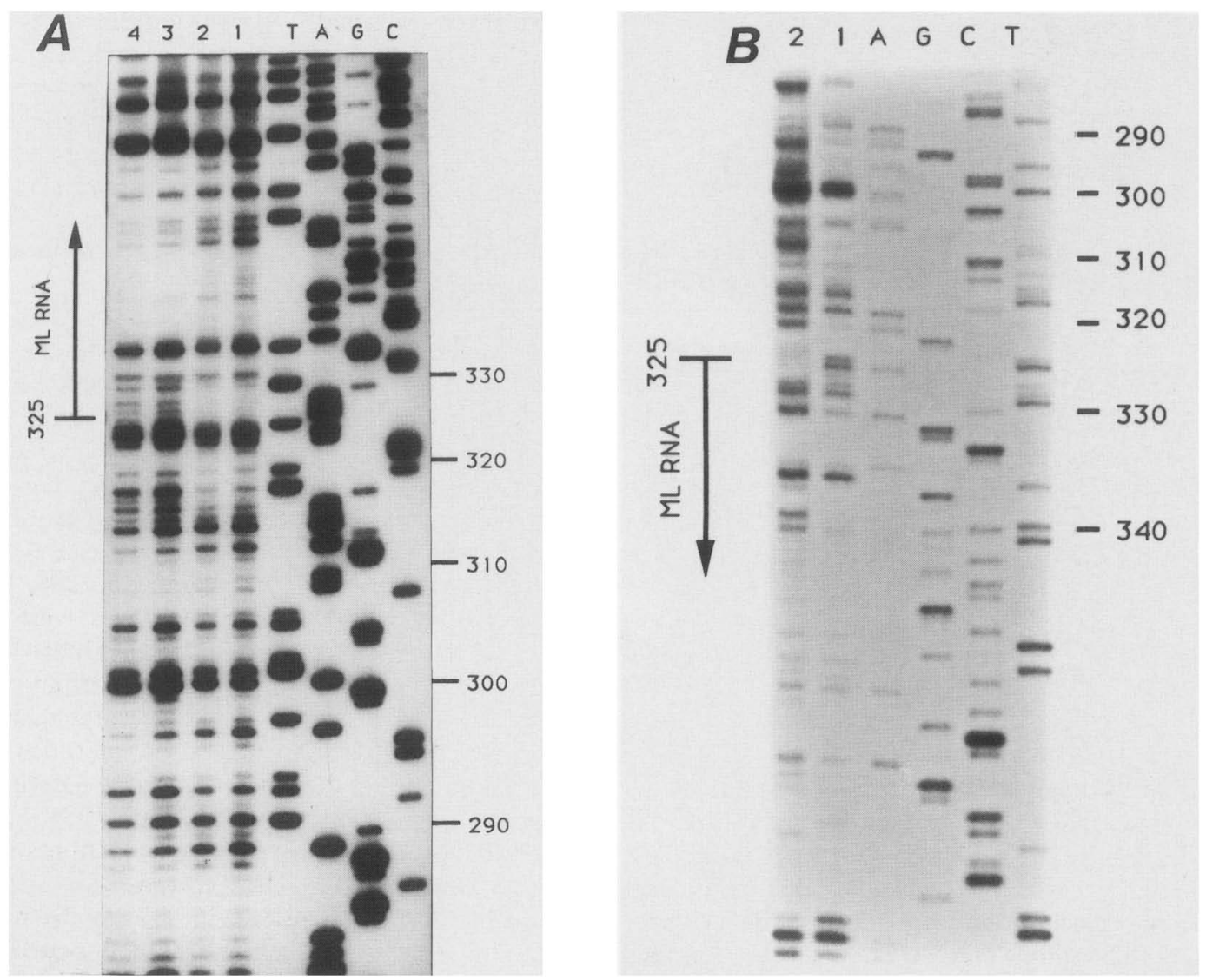

Figure 4. $(A)$ MNase digestion pattern of the late mRNA template strand at the major late promoter. Permeabilized SV40-infected cells were treated with $250 \mathrm{U}$ (lane 2) or $500 \mathrm{U}$ (lane 1). In vitro naked DNA was treated with $0.01 \mathrm{U}$ (lane 4) or $0.025 \mathrm{U}$ (lane 3) of MNase in total volume of $200 \mu l$ of solution C. The samples were probed with primer 260 . Sequences were obtained by dideoxy sequencing. $(B)$ MNase digestion pattern of the SV40 major late promoter region in lysolecithin-permeabilized cells. SV40 DNA in permeabilized cells and in vitro naked DNA were digested with MNase and then probed with primer 404. Permeabilized SV40-infected cells were treated with $500 \mathrm{U}$ of MNase (lane 2). In vitro naked DNA was treated with $0.025 \mathrm{U}$ (lane 1) of MNase in a total volume of $200 \mu \mathrm{l}$ of solution C.

and Gralla 1988, 1989; O'Halloran et al. 1989|. Thus, we attempted to adapt $\mathrm{KMnO}_{4}$ probing to SV40-infected CV-1 cells.

\section{$\mathrm{KMnO}_{4}$ probing of melted DNA}

Because $\mathrm{KMnO}_{4}$ probing of Escherichia coli involves adding the reagent directly to growing cells, the chemical was applied to CV-l monolayers without prior permeabilization (see Fig. 1). The SV40 DNA was isolated and the $\mathrm{KMnO}_{4}$ modification pattern was revealed by probing with primer 404; recall that this is the primer that, when used as a probe for MNase digestion, pointed to backbone exposure. The $\mathrm{KMnO}_{4}$ attack pattern is shown in Figure 5, lane 4. This can be compared with the in vitro modification pattern shown in lane 3 . The two patterns are very similar except that bands in the middle region are slightly more reactive in situ. Comparable experiments probing the termination region, as in Figure 2, did not show such enhanced reactivity /data not shown).

$\mathrm{KMnO}_{4}$ selectivity for melted DNA may be increased by lowering the temperature, and therefore, the experi- ment was repeated but the monolayer was placed on ice prior to treatment with $\mathrm{KMnO}_{4}$ (the naked DNA pattern is unchanged at $0^{\circ} \mathrm{C}$ ). The resulting modification pattern in situ is shown in lane 2 . Comparing lane 2 with either the in vitro lanes 3 and 1 or the $37^{\circ} \mathrm{C}$ in situ lane 4 , it can be seen that the slight hyperreactivity is enhanced, particularly in this central region. Essentially, every band in the region from approximately the transcription start site at nucleotide 325 back to nucleotide 297 is somewhat hyperreactive to $\mathrm{KMnO}_{4}$ attack (lane 2). Densitometry indicates that the hyperreactivity is about four- to fivefold, compared to naked DNA. Other isolated bands in the upper part of the autoradiogram that are hypersensitive are near minor transcriptional start sites. $\mathrm{KMnO}_{4}$ probing in permeabilized cells at $37^{\circ} \mathrm{C}$ and $0^{\circ} \mathrm{C}$ gave similar results (data not shown).

The $\mathrm{KMnO}_{4}$ hyperreactive region from nucleotide 297 to approximately nucleotide 330 is within the MNase hyperreactive region on the same DNA strand. $\mathrm{KMnO}_{4}$ probing of the opposite strand showed only weak hyperreactivity (data not shown), consistent with the lack of MNase hypersensitivity on that strand. Asymmetric at- 


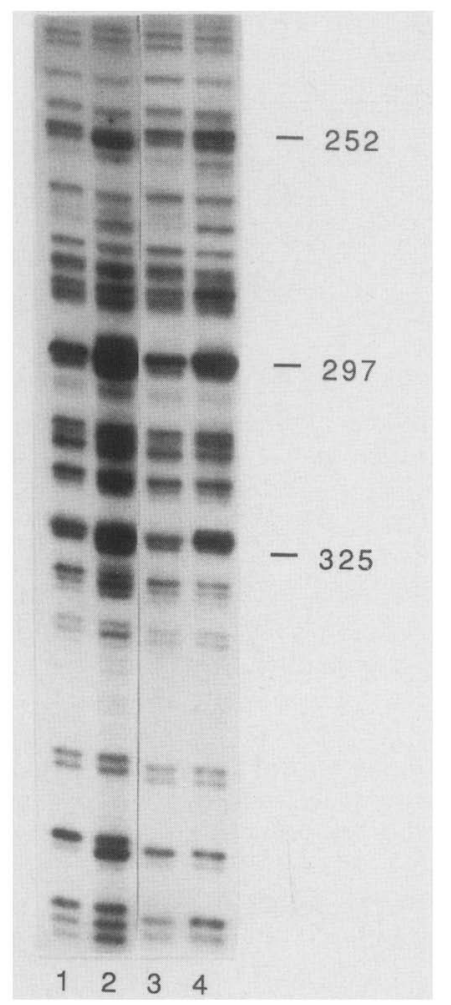

Figure 5. $\mathrm{KMnO}_{4}$ modification pattern of the SV40 late promoter region in intact cells. SV40 DNA was probed with primer 404. SV40-infected cells were treated with $\mathrm{KMnO}_{4}$ at $37^{\circ} \mathrm{C}(20$ $\mathrm{mM}$, lane 4$)$ or at $0^{\circ} \mathrm{C}\left(150 \mathrm{~mm} \mathrm{KMnO}_{4}\right.$, lane 2). As a control, purified SV40 DNA was treated with $\mathrm{KMnO}_{4}$ at $37^{\circ} \mathrm{C}$ for $4 \mathrm{~min}$ (10 mM, lane 1; $5 \mathrm{~mm}$, lane 3) and quenched with $\beta$-mercaptoethanol.

tack in open promoter DNA has also been seen in bacteria (Sasse-Dwight and Gralla 1988). The hyperreactive SV40 strand is the nontemplate DNA strand, whereas the template strand is less reactive, probably because it is protein-bound, as suggested by its protection from MNase. Because MNase reacts with preference toward single-stranded DNA and $\mathrm{KMnO}_{4}$ has an even stronger single strand preference, the DNA in this region appears to be melted. We explored the relationship of this melting to transcription by probing after pretreating cells with either of the RNA polymerase II inhibitors $\alpha$ amanitin or rifamycin AF-013 (Meilhac et al. 1972; Crerar and Pearson 1977). The latter proved too toxic to cells to yield reliable data, but the former showed little effect, consistent with its role as an inhibitor of elongation rather than initiation. The protection of the template strand may indicate that a factor binds the region containing the start site and holds the template strand closely and the nontemplate strand loosely. All of these interactions are with DNA elements known to be critical for transcription from the major late promoter.

\section{Discussion}

We developed and applied new in situ probing methods to investigate the nucleoprotein structure that involves the three proximal transcription elements of the SV40 major late promoter at $40 \mathrm{hr}$ post-infection. Lysolecithin permeabilization, shown previously to allow continued initiation of transcription from this promoter (Contreras and Fiers 1981), was shown to promote entry of two nucleases that can probe the promoter structure. Additionally, potassium permanganate was used to probe intact cells for melted promoter DNA, on the basis of a procedure used for detecting such DNA in bacterial cells (Sasse-Dwight and Gralla 1988, 1989). Collectively, this allowed in situ probing with three different reagents: DNase I, which recognizes and cleaves from the DNA minor groove (Drew 1984; Suck et al. 1988), micrococcal nuclease, which recognizes and cleaves the DNA backbone directly (Cockell et al. 1983; Drew 1984) and $\mathrm{KMnO}_{4}$, which recognizes and oxidizes melted pyrimidines preferentially (Hayatsu and Ukita 1967; Fritzsche et al. 1987; Sasse-Dwight and Gralla 1988, 1989). In this study, the reagent cleavage patterns were detected by primer-extension methods and facilitated by probing viral DNA, which is present in multiple extrachromosomal copies. In principle, however, the reagent cleavage pattern could be detected by other methods, for example by footprinting procedures involving blotting to cleaved genomic DNA (Church and Gilbert 1984; Ephrussi et al. 1985). Thus, the new probing procedures could potentially have widespread applicability.

The results of applying these new methods to probe the SV40 major late promoter are shown in Figure 6, A and $B$, which summarizes the probing results and a proposed nucleoprotein structure, respectively. This figure shows only the DNA surrounding the transcription start site at nucleotide 325 , which can be subdivided into three transcription control elements /see the bars in the middle of the sequence) (Brady et al. 1982; Ayer and Dynan 1988); all of these elements appear to be included in nucleoprotein complexes. The downstream element is centered near nucleotide 353 in the transcribed region. The DNA within this element shows DNase I hypersensitivity at 10- to $11-\mathrm{bp}$ intervals, indicating that it is part of a complex that involves wrapped DNA (Chao et al. 1979; Simpson and Stafford 1983; Drew and Travers 1985; Sawadogo and Roeder 1985; Hochschild and Ptashne 1986; Zinn and Maniatis 1986). Because these periodic sites continue beyond the element and cover a nucleosome-size region, we strongly suspect that the element is bound by a specifically positioned nucleosome, probably centered near nucleotide 395 .

The two remaining transcription control elements, corresponding approximately to a 40-bp region from nucleotide 290 to 330 (see Fig. 6A), also appear to be part of a specific nucleoprotein complex. The template DNA strand is protected against micrococcal nuclease attack, which indicates factor binding. This bound region begins at nucleotide 330 just beyond the start site and continues to nucleotide 316 . On the nontemplate strand, the protection is highly localized to a few nucleotides surrounding the start site. Most of the sequences in the proximal elements on the nontemplate strand are, by contrast, hyperreactive to attack by micrococcal nuclease and $\mathrm{KMnO}_{4}$. Because both reagents react preferen- 

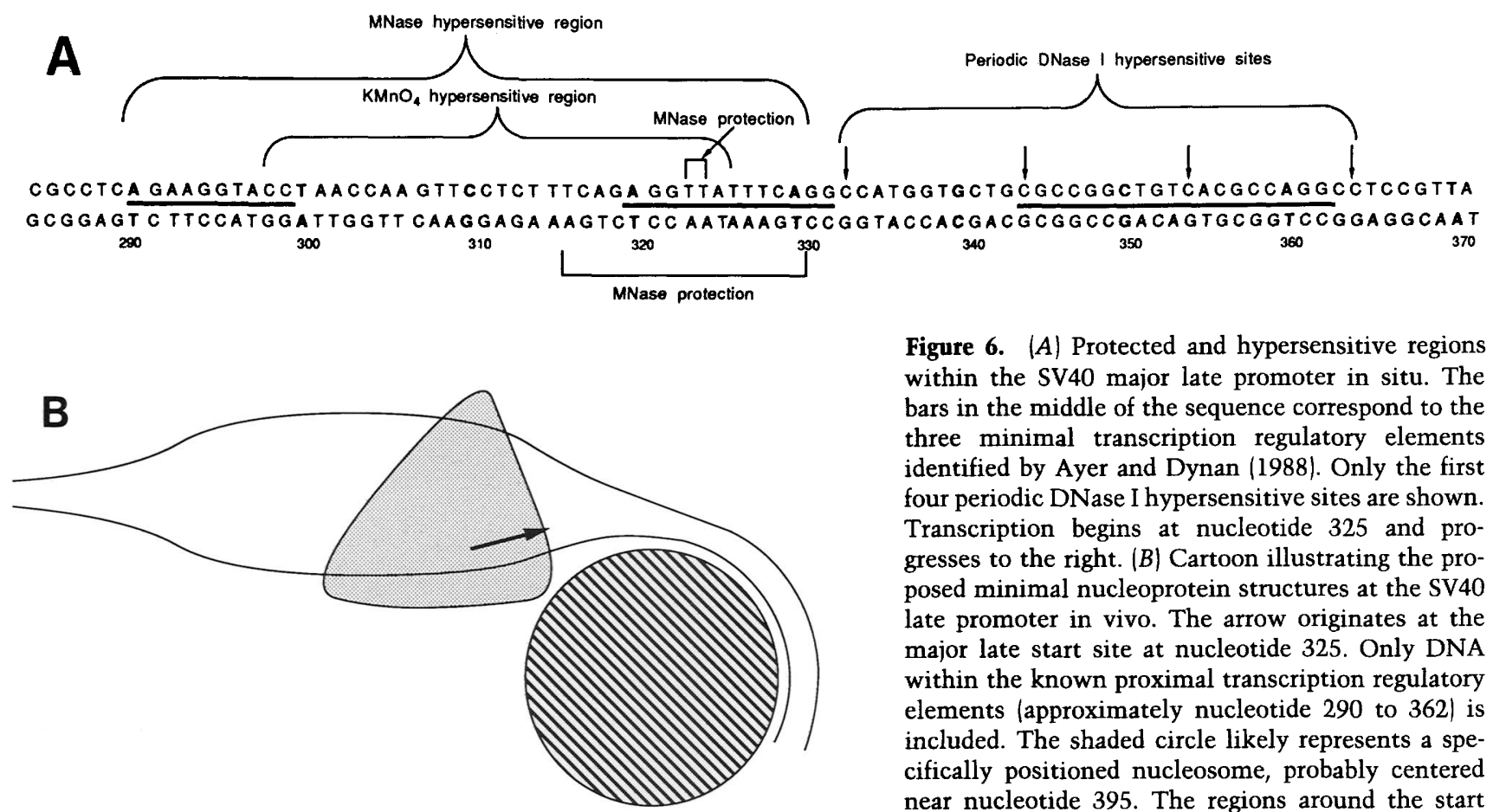

Figure 6. (A) Protected and hypersensitive regions within the SV40 major late promoter in situ. The bars in the middle of the sequence correspond to the three minimal transcription regulatory elements identified by Ayer and Dynan (1988). Only the first four periodic DNase I hypersensitive sites are shown. Transcription begins at nucleotide 325 and progresses to the right. $(B)$ Cartoon illustrating the proposed minimal nucleoprotein structures at the SV40 late promoter in vivo. The arrow originates at the major late start site at nucleotide 325 . Only DNA within the known proximal transcription regulatory elements (approximately nucleotide 290 to 362 ) is included. The shaded circle likely represents a specifically positioned nucleosome, probably centered near nucleotide 395 . The regions around the start site that are covered by the shaded wedge represent bound protecting factors. These structures represent only those that predominate in situ; limiting and non-DNA binding factors are not detectable by current methods.

tially with single-stranded DNA, and because $\mathrm{KMnO}_{4}$ hyperreactivity marks polymerase-melted DNA at bacterial promoters (Sasse-Dwight and Gralla 1988, 1989; O'Halloran et al. 1989), we believe that these sequences are melted and exposed (Fig. 6B).

We believe that the structures indicated by these new probing methods are part of the biologically relevant transcription machinery. As mentioned above, lysolecithin permeabilization is much gentler than isolation of nuclei, and therefore, such processes as initiation of transcription and DNA replication can occur (Contreras and Fiers 1981). The physiological relevance of these bound, melted and wrapped DNA structures is supported further by their association with critical transcription elements. Moreover, the limits of the melted DNA region are analogous to the limits in bacterial transcription complexes (Siebenlist et al. 1980). The downstream border of melting is similar in the two cases, just beyond the transcription start point; this is of course necessary to copy the DNA by exposing the basepairing positions. The upstream border in $E$. coli is within the TATA-like element near -10 (Siebenlist et al. 1980; Sasse-Dwight and Gralla 1989). The upstream border at the SV40 promoter is within the position occupied by the upstream proximal element near nucleotide 297. The majority of polymerase II promoters have a TATA element in this position.

Although these structures are very likely biologically important, their roles in transcriptional regulation cannot be deduced from these studies. The nucleases probe primarily those SV40 templates that are not encapsidated with coat proteins. These templates constitute a minority of the SV40 intracellular DNA, but in turn, only a minority of these nonencapsidated templates are believed to be transcriptionally active (see $\mathrm{Bu}$ chanan and Gralla 1987). The strength of the nuclease digestion patterns suggests that a majority of the nonencapsidated DNA participates in the structures illustrated in Figure 6B. Therefore, these structures probably typify the state of the major late promoter DNA in vivo, but one cannot state definitively whether they are involved in transcriptional activation or repression. Clarification of their roles must await reconstitution of the physiologically appropriate transcription apparatus. Such a system will surely require factors not seen in this study, both limiting factors that are bound to too few templates to allow detection of protection and nonDNA-binding factors. However, the structures indicated by these in situ probing studies should serve to guide the reconstruction of the physiologically appropriate transcription apparatus.

The existence of a specifically positioned nucleosome on the downstream transcription element is rather unexpected because most nucleosomes are not uniquely positioned (although some do occur on active genes: McGhee et al. 1981; Simpson and Stafford 1983; Sawadogo and Roeder 1985; De Bernardin et al. 1986). Although DNA sequence can in some cases dictate nucleosome positioning (Chao et al. 1979), this is apparently not the case here because we have shown 
previously (Poljak and Gralla 1987, but see Mengeritsky and Trifonov 1984| that reconstituted nucleosomes do not form preferentially with this SV40 sequence. Therefore, it is very likely that the positioning of the nucleosome just downstream from the transcription start site depends on interactions with nearby factors. One obvious candidate for such a positioning factor is the factor or factors that bind the adjacent transcription element. Such an interaction would mean that the positioned SV40 nucleosome was interacting with a transcription factor bound to the start site where RNA polymerase II must be located. Baer and Rhodes (1983) showed that RNA polymerase II can bind to modified nucleosome core particles in transcribed regions. Therefore, it is possible that the three DNA elements are part of a single large nucleoprotein complex that contains the factors illustrated in Figure 6B as well as RNA polymerase II and other factors.

Analogous specialized nucleoprotein structures that involve multiple protein-protein interactions and DNA-protein interactions have been shown to be crucial for regulated replication and expression of the genome in prokaryotic systems (see Echols 1986). The anatomy of the complex at the SV40 major late promoter has strong similarities to $E$. coli regulatory complexes. First, in both the E. coli promoter and the eukaryotic SV40 major late polymerase II promoter, the DNA is melted from the TATA-box position to the initiation site. Second, the overall structure is similar to the prepriming complex for replication initiation at the $E$. coli chromosome (Bramhill and Kornberg 1988) and also to the nucleoprotein complexes formed on E. coli malEpmalKp promoter (Raibaud et al. 1989) and to some extent the lac promoter (Zhang and Gralla 1989); wrapped nucleoprotein particles appear to be involved in all these complexes. An important function of the bacterial complexes is to assist in melting the DNA to allow it to be copied in replication and transcription. All the complexes have multiple DNA elements specifying the interaction of multiple proteins. These experiments on the SV40 major late promoter also indicate the existence of a higher order nucleoprotein complex that contains multiple DNA elements and melted DNA, in this case at a mammalian polymerase II promoter. It appears that specifically positioned wrapped nucleoprotein complexes (Echols 1986) may represent a common way to mark starts of DNA transcription as well as DNA replication.

\section{Materials and methods}

\section{Materials}

Primer 404 (ACAAAAAGGCGTTGAGCTGT), which hybridizes to the early mRNA template strand from nucleotide 404 to 423 , primer 489 (TTCAGTTAACCTTTCTGGTT), which hybridizes to the early mRNA template strand from 489 to 508, primer 260 (TCCACACCCTAACTGACA), which hybridizes to the late mRNA template strand from 242 to 260 , and primer 2556 (GACATGATAAGATACAT), which hybridizes to the late mRNA template strand from 2540 to 2556 , were synthesized at the UCLA facility. Micrococcal nuclease and deoxyribonuclease I were purchased from Worthington Biochemical
Corp.; Klenow fragment was purchased from Bethesda Research Laboratories. Lysolecithin ( $L-\alpha$-lysophosphatidylcholine, type I) was purchased from Sigma.

Treatment of lysolecithin-permeabilized cells with DNase I and MNase

In general, CV-1 cells were infected with 1-10 pfu per cell wild-type SV40 strain 776 and maintained in Dulbecco's modified Eagle's medium (DMEM) plus $2 \%$ calf serum in 10 -cm-diameter plates. At $40 \mathrm{hr}$ postinfection, cells held at $37^{\circ} \mathrm{C}$ were treated with $0.5 \mathrm{mg} / \mathrm{ml}$ of lysolecithin in solution A [150 mM sucrose, $80 \mathrm{mM} \mathrm{KCl}, 35 \mathrm{~mm}$ HEPES ( $\mathrm{pH} 7.4$ ), $5 \mathrm{~mm}$ potassium phosphate, $5 \mathrm{mM} \mathrm{MgCl}_{2}, 0.5 \mathrm{mM} \mathrm{CaCl}_{2}$ ] for $1 \mathrm{~min}$ at $37^{\circ} \mathrm{C}$ (Contreras and Fiers 1981). Then this lysolecithin solution was removed by aspiration. Then cells were washed with solution $\mathrm{A}$ and $1 \mathrm{ml}$ of DNase $I(\sim 0.1 \mathrm{mg} / \mathrm{ml})$ in solution B (solution A with $80 \mathrm{~mm}$ of $\mathrm{NaCl}$ instead of $\mathrm{KCl}$ and with $2 \mathrm{mM} \mathrm{CaCl})_{2}$, or MNase $(250-500 \mathrm{U} / \mathrm{ml})$ in solution C [ $150 \mathrm{~mm}$ sucrose, $50 \mathrm{mM}$ Tris- $\mathrm{HCl}$ ( $\mathrm{pH} \mathrm{7.5}$, $50 \mathrm{mM} \mathrm{NaCl}$, and $2 \mathrm{mM} \mathrm{CaCl}_{2}$ ] was then added for $2 \mathrm{~min}$ at $37^{\circ} \mathrm{C}$. The enzyme solution was aspirated off the plates, and Hirt lysis solution (Hirt 1967) was added immediately to quench the residual DNase I or MNase activity. Finally, SV40 DNA was purified and probed as described (Buchanan and Gralla 1987).

\section{Treatment of intact SV40-infected CV-1 cells with $\mathrm{KMnO}_{4}$}

At $40 \mathrm{hr}$ postinfection, CV-1 cells were washed with phosphate-buffered saline (PBS). $\mathrm{KMnO}_{4}\left(20 \mathrm{mM}\right.$ at $37^{\circ} \mathrm{C}, 150 \mathrm{~mm}$ at $0^{\circ} \mathrm{Cl}$ in PBS was added. The cells were then scraped off the plates and spun down in a microcentrifuge, the entire procedure taking 1-1.5 minutes. The supernatant was removed and the residual $\mathrm{KMnO}_{4}$ was quenched with $280 \mu \mathrm{l}$ of $1.4 \mathrm{M} \beta$-mercaptoethanol. Then $720 \mu 1$ of $0.84 \%$ SDS $+14 \mathrm{mM}$ EDTA (pH 7.5) was added (Hirt 1967), and the SV40 DNA was purified and probed as described (Buchanan and Gralla 1987).

\section{Primer extension and gel electrophoresis}

The sites of DNase I or MNase nicking or $\mathrm{KMnO}_{4}$ modification were determined by the hybridization of primers labeled at the $5^{\prime}$ end with $\left[\gamma^{-32}\right]$ ATP and T4 polynucleotide kinase to denatured samples and extension to the sites with nicks or modification as described (Gralla 1985; Buchanan and Gralla 1987; Sasse-Dwight and Gralla 1989). There was minimal background banding in the absence of added nuclease or $\mathrm{KMnO}_{4}$. Briefly, about $300,000 \mathrm{cpm}$ of end-labeled primer was added and followed by $\mathrm{NaOH}$ to bring the $\mathrm{pH}$ to 11 . The DNA was denatured by heating to $80^{\circ} \mathrm{C}$ for $2 \mathrm{~min}$. After neutralization, the primers were hybridized at $45^{\circ} \mathrm{C}$ for $3 \mathrm{~min}$ and extended with Klenow DNA polymerase at $50-52^{\circ} \mathrm{C}$ for $10 \mathrm{~min}$. The DNA samples were then precipitated with ethanol and analyzed by $6 \%$ (wt/ vol) acrylamide $/ 50 \%$ (wt/vol) urea DNA sequencing gels.

\section{Acknowledgments}

This research was supported by grant DMB85-12033 from the National Science Foundation.

\section{References}

Ayer, D.E. and W.S. Dynan. 1988. Simian virus 40 major late promoter: A novel tripartite structure that includes intragenic sequences. Mol. Cell Biol. 8: 2021-2033.

Baer, W.B. and D. Rhodes. 1983. Eukaryotic RNA polymerase II 
binds to nucleosome cores from transcribed genes. Nature 301: 482-488.

Brady, J., M. Radonovich, M. Vodkin, V. Natarajan, M. Thoren, G. Das, J. Janik, and N.P. Salzman. 1982. Site-specific basic substitution and deletion mutations that enhance or suppress transcription of the SV40 major late RNA. Cell 31: 626-633.

Bramhill, D. and A. Kornberg. 1988. Duplex opening by dnaA protein at novel sequences in initiation of replication at the origin of the E. coli chromosome. Cell 52: 743-755.

Buchanan, R.L. and J.D. Gralla. 1987. Factor interactions at simian virus $40 \mathrm{GC}$-box promoter elements in intact nuclei. Mol. Cell Biol. 7: 1554-1558.

Buratowski, S., S. Hahn, L. Guarente, and P.A. Sharp. 1989. Five intermediate complexes in transcription initiation by RNA polymerase II. Cell 56: $549-561$.

Chao, M.V., J.D. Gralla, and H.G. Martinson. 1979. DNA sequence directs placement of histone cores on restriction fragments during nucleosome formation. Biochemistry 18: $1068-1074$.

Church, G.M. and W. Gilbert. 1984. Genomic sequencing. Proc. Natl. Acad. Sci. 81: 1991-1995.

Cockell, M., D. Rhodes, and A. Klug. 1983. Location of the primary sites of micrococcal nuclease cleavage on the nucleosome core. J. Mol. Biol. 170: 423-446.

Contreras, R. and W. Fiers. 1981. Initiation of transcription by RNA polymerase II in permeable SV40-infected CV-1 cells; evidence for multiple promoters of SV40 late transcription. Nucleic Acids Res. 2: 215-236.

Crerar, M.M. and M.L. Pearson. 1977. RNA polymerase II regulation in $\alpha$-amanitin-resistant rat myoblast mutants. Changes in wild-type and mutant enzyme levels during growth in $\alpha$-amanitin. J. Mol. Biol. 112: 331-342.

De Bernardin, W., T. Koller, and J.M. Sogo. 1986. Structure of in vivo transcribing chromatin as studied in simian virus 40 minichromosome. J. Mol. Biol. 191: 469-482.

Dieters, J.A., J.C. Gallucci, and R.R. Holms. 1982. Computer simulation of staphylococcal nuclease action on thymidine 3',5'-Bis(phosphate)(pdTp). J. Am. Chem. Soc. 104: $5457-$ 5465.

Dingwall, C., G.P. Lomonossof, and R.A. Lasky. 1981. High sequence specificity of micrococcal nuclease. Nucleic Acids Res. 9: 2659-2673.

Dorn, A., J. Bollekens, A. Staub, C. Benoist, and D. Mathis. 1987. A multiplicity of CAAT box-binding proteins. Cell 50: $863-872$.

Drew, H.R. 1984. Structural specificities of five commonly used DNA nucleases. I. Mol. Biol. 176: 535-557.

Drew, H.R. and A.A. Travers. 1985. DNA bending and its relation to nucleosome positioning. I. Mol. Biol. 186: 773-790.

Echols, H. 1986. Multiple DNA-protein interactions governing high-precision DNA transactions. Science 233: 1050-1056.

Ephrussi, A., G.M. Church, S. Tonegawa, and W. Gilbert. 1985. B Lineage-specific interactions of an immunoglobulin enhancer with cellular factors in vivo. Science 227: 134-140.

Fritzsche, E., H. Hayatsu, G.L. Igloi, S. Iida, and H. Kössel. 1987. The use of permanganate as a sequencing reagent for identification of 5-methylcytosine residues in DNA. $\mathrm{Nu}$ cleic Acids Res. 15: 5517-5528.

Galas, D. and A. Schmitz. 1978. DNase footprinting: A simple method for the detection of protein-DNA binding specificity. Nucleic Acids Res. 5: 3157-3170.

Gralla, J.D. 1985. Rapid 'footprinting' on supercoiled DNA. Proc. Natl. Acad. Sci. 82: 3078-3081.

Griffith, J.D. 1975. Chromatin structure: Deduced from a minichromosome. Science 187: 1202-1203.
Hayatsu, H. and T. Ukita. 1967. The selective degradation of pyrimidines in nucleic acids by permanganate oxidation. Biochem. Biophys. Res. Commun. 29: 556-561.

Hirt, B. 1967. Selective extraction of polyoma DNA from infected mouse cell cultures. J. Mol. Biol. 26: 365-369.

Hochschild, A. and M. Ptashne. Cooperative binding of $\lambda$ repressors to sites separated by integral turns of the DNA helix. Cell 44: 681-687.

Lutter, L. 1978. Kinetic analysis of DNase I cleavages in the nucleosome core: Evidence for a DNA superhelix. I. Mol. Biol. 124: 391-420.

McGhee, J.D. and G. Felsenfeld. 1983. Another potential artifact in the study of nucleosome phasing by chromatin digestion with micrococcal nuclease. Cell 32: 1205-1215.

McGhee, J.D., W.I. Wood, M. Dolan, J.D. Engel, and G. Felsenfeld. 1981. A 200 base pair region at the $5^{\prime}$ end of the chicken adult $\beta$-globin gene is accessible to nuclease digestion. Cell 27: 45-55.

Meilhac, M., Z. Tysper, and P. Chambon. 1972. Animal DNAdependent RNA polymerase. 4. Studies on inhibition by rifamycin derivatives. Eur. J. Biochem. 28: 291-300.

Mengeritsky, G. and E.N. Trifonov. 1984. Nucleotide sequence-directed mapping of the nucleosome of SV40 chromatin. Cell Biophys, 6: 1-8.

O'Halloran, T.V., B. Franz, M.K. Shin, D.M. Ralson, and J.G. Wright. 1989. The MeR heavy metal receptor mediates positive activation on a topologically novel transcription complex. Cell 56: 119-128.

Piette, J., S. Cereghini, M.-H. Kryszke, and M. Yaniv. 1986. Identification of cellular proteins that interact with polyoma virus or simian virus 40 enhancers. Cancer Cells 4: $103-113$.

Poljak, L. and J.D. Gralla. 1987. Competition for formation of nucleosomes on fragmented SV40 DNA: A hyperstable nucleosome forms on the termination region. Biochemistry 26: $295-303$.

Raibaud, O., D. Vidal-Ingigliardi, and E. Richet. 1989. A complex nucleoprotein structure involved in activation of transcription of two divergent Escherichia coli promoters. I. Mol. Biol. 205: 471-485.

Sasse-Dwight, S. and J.D. Gralla. 1988. Probing the Escherichia coli gInALG upstream activation mechanism in vivo. Proc. Natl. Acad. Sci. 85: 8934-8938.

- 1989. $\mathrm{KMnO}_{4}$ as a probe for lac promoter DNA melting and mechanism in vivo. J. Biol. Chem. 264: 8074-8081.

Sawadogo, M. and R.G. Roeder. 1985. Interaction of a gene-specific transcription factor with the adenovirus major late promoter upstream of the TATA box region. Cell 43: 165-175.

Shelton, E., P.M. Wassarmann, and M.L. DePamphilis. 1978. Structure, spacing and phasing of nucleosomes on isolated forms of mature simian virus 40 chromosomes. /. Biol. Chem. 255: 771-782.

Siebenlist, U., R.B. Simpson, and W. Gilbert. 1980. E. coli RNA polymerase interacts homologously with two different promoters. Cell 20: 269-281.

Simpson, R.T. and D. W. Stafford. 1983. Structural features of a phased nucleosome core particle. Proc. Natl. Acad. Sci. 80: $51-55$

Suck, D., A. Lahm, and C. Oetner. 1988. Structure refined to $2 \AA$ of a nicked DNA octanucleotide complex with DNase I. Nature 332: 464-468.

Zhang, L. and J.D. Gralla. 1989. Micrococcal nuclease as a probe for bound and distorted DNA in lac transcription and repression complexes. Nucleic Acids Res. 17: 5017-5028.

Zinn, K. and T. Maniatis. 1986. Detection of factors that interact with the human $\beta$-interferon regulatory region in vivo by DNase I footprinting. Cell 45: 611-618. 


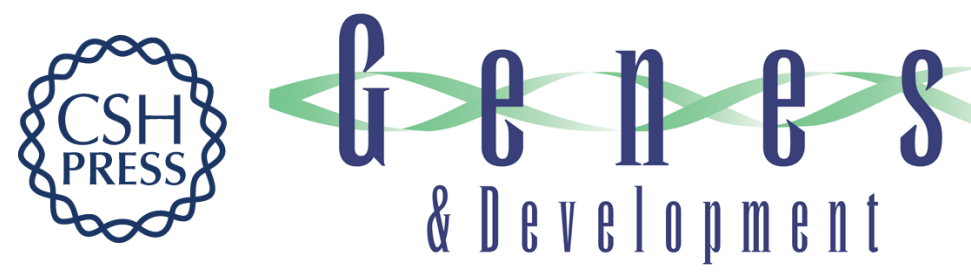

\section{In situ nucleoprotein structure at the SV40 major late promoter: melted and wrapped DNA flank the start site.}

L Zhang and J D Gralla

Genes Dev. 1989, 3:

Access the most recent version at doi:10.1101/gad.3.11.1814

References This article cites 43 articles, 10 of which can be accessed free at:

http://genesdev.cshlp.org/content/3/11/1814.full.html\#ref-list-1

License

Email Alerting

Service

Receive free email alerts when new articles cite this article - sign up in the box at the top right corner of the article or click here.

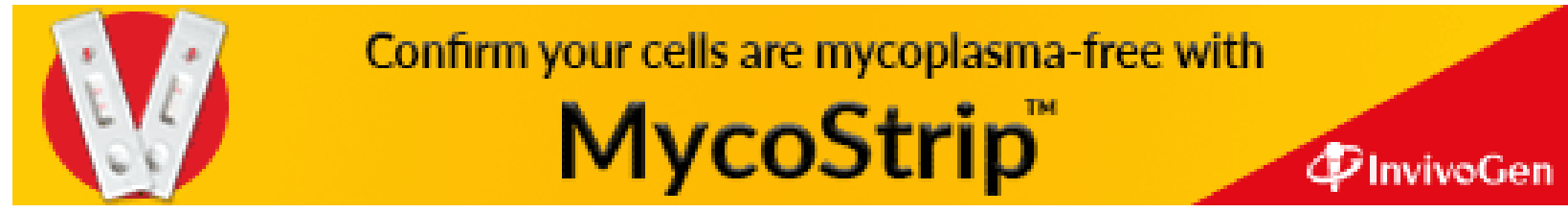

